\title{
High expression of substance $P$ and its receptor neurokinin-I receptor in colorectal cancer is associated with tumor progression and prognosis
}

This article was published in the following Dove Press journal:

OncoTargets and Therapy

16 June 2016

Number of times this article has been viewed

Xiao-Yi Chen, ',* Guo-Qing $\mathrm{Ru},{ }^{2, *}$ Ying-Yu Ma, ' Jun Xie, ${ }^{3}$ Wan-Yuan Chen, ${ }^{2}$ Hui-Ju Wang,' Shi-Bing Wang,' Li Li,' Ke-Tao Jin, ${ }^{4}$ Xiang-Lei $\mathrm{He},{ }^{2}$ Xiao-Zhou Mou'

'Clinical Research Institute, ${ }^{2}$ Department of Pathology, Zhejiang Provincial People's Hospital, Hangzhou, ${ }^{3}$ Department of AnusIntestines, Affiliated Hospital of Shaoxing University, ${ }^{4}$ Department of Gastrointestinal Surgery, Shaoxing People's Hospital, Shaoxing Hospital of Zhejiang University, Shaoxing, People's Republic of China

*These authors contributed equally to this work

\begin{abstract}
Background: Epidemiologic evidence suggests that chronic inflammation and/or chronic infection is associated with cancer development, and the inflammatory process may play a crucial role in the carcinogenesis and prognosis of colorectal cancer (CRC). Substance P (SP) belongs to the family of tachykinins and acts as an immunomodulator, binding to the neurokinin-1 receptor (NK1R) to initiate tumor cell proliferation, angiogenesis, and migration, steps that are critical for tumor cell invasion and metastasis. It is suggested that SP/NK1R signaling may play an important role in cancer progression and metastasis. However, the exact involvement and significance of SP and NK1R in CRC pathologies remain to be adequately deciphered.
\end{abstract}

Patients and methods: We performed immunohistochemistry staining on tissue microarrays containing 267 pairs of $\mathrm{CRC}$ and adjacent normal tissues to evaluate the clinical significance of SP or NK1R in the progression and prognosis of CRC. We also explored the potential correlation between SP and NK1R in CRC development.

Results: Expression levels of SP and NK1R were upregulated in CRC compared with their expressions in adjacent normal tissues $(P<0.001)$. High expression of SP in CRC was significantly associated with lymph node metastasis $(P<0.001)$. We also found that high expression of NK1R in CRC was significantly related to TNM (tumor node metastasis) stage $(P=0.010)$ and lymph node metastasis $(P=0.019)$. A high correlation between SP and NK1R expression was also observed $(r=0.419, P<0.001)$. Survival analysis showed that $\mathrm{CRC}$ patients with high expression of SP or NK1R have a poor prognosis when compared to patients with low SP or NK1R expression (log rank test, $P<0.05)$. Multivariate analysis using Cox regression model showed that survival was independently correlated with lymph node metastasis, distant metastasis, and SP expression $(P<0.05)$.

Conclusion: Upregulation of SP-NK1R may play a crucial role in CRC progression. Moreover, SP-NK1R expression may also be used as a predictor for CRC prognosis.

Keywords: SP, NK1R, CRC, progression, prognosis

\section{Introduction}

Colorectal cancer (CRC) is the fourth leading cause of cancer-related deaths in the world, with increasing mortality observed in recent years. ${ }^{1,2}$ In the People's Republic of China, CRC is one of the eight most common forms of malignancy. ${ }^{3}$ Despite advances in clinical research and care, as well as knowledge regarding molecular mechanisms involved in CRC, relatively poor prognosis and high death rates are major challenges for $\mathrm{CRC}$ treatment. Moreover, the mechanisms associated with the pathogenesis of CRC are still not completely defined. Development of CRC is thought to begin with accumulated genetic changes in a cell such as point mutations, deletions and amplification, and methylation of DNA, leading to irreversible genetic damage. ${ }^{4}$ 
Recently, many studies have demonstrated that mediators of inflammation can induce DNA damage and lead to initiation and progression of cancer. ${ }^{5}$ Evidence also suggests that chronic inflammation and/or chronic infection is associated with cancer development in various types of tumor, including the development of CRC. ${ }^{5}$

Tachykinins include an evolutionary conserved family of peptide hormones that are potent immunomodulators and have been shown to have implications in the process of carcinogenesis. It is known that substance $\mathrm{P}$ (SP), neurokinin A (NKA), and neurokinin B (NKB) belong to the tachykinin family as proinflammatory neuropeptides. ${ }^{6,7} \mathrm{SP}$ and NKA are derived from the preprotachykinin A gene, whereas NKB is derived from the preprotachykinin $\mathrm{B}$ gene. The biological actions of SP, NKA, and NKB are mediated by receptors named neurokinin-1 receptor (NK1R), neurokinin-2 receptor, and neurokinin-3 receptor. SP is synthesized by various cells, regulating biological processes such as exocrine and endocrine secretion, connective tissue proliferation, and blood pressure regulation. ${ }^{8,9}$ Most importantly, SP plays a role in regulating neurogenic inflammation and immune responses in peripheral tissues and the central nervous system. ${ }^{10,11}$ The receptor subtype NK1R has the highest activity for SP and has been identified in many cancer subtypes and can mediate various physiologic and pathophysiologic responses, including cell proliferation, migration, and inflammation. ${ }^{12-17}$ Studies have demonstrated that expression levels of functional NK1R in tumor are associated with the degree of malignancy of those tumors. ${ }^{16,18}$ Binding of SP to NK1R initiates tumor cell proliferation, angiogenesis, and migration, which are critical for tumor cell invasion and metastasis, implicating an important role of SP/NK1R signaling in cancer progression and metastasis. ${ }^{19-22}$ Researchers found that in tumor tissues the NK-induced signaling is altered when compared to normal cells so as to support proliferation and survival of cancer cells. Our previous study suggested that $N K 1 R$ polymorphism alone or in combination with $N K 2 R$ is a promising prognostic marker of lymph node metastasis in CRC patients. ${ }^{23}$ However, the exact signaling cascades involved and the role of $\mathrm{SP}$ and NK1R in CRC pathologies remain to be elucidated.

In order to pin the role of inflammatory processes in CRC, here, we evaluate the expression of SP and NK1R and its clinical significance in CRC patients to explore the potential association between SP and NK1R in CRC progression and prognosis. We expect that understanding the involvement of inflammatory processes in $\mathrm{CRC}$, in combination with any other genetic factors, could be used as a tool to reduce the incidence of CRC and for better clinical management of the disease.

\section{Patients and methods CRC patients}

The present study was approved by the Ethics Committee of Zhejiang Provincial People's Hospital (Hangzhou, Zhejiang, China). All patients provided written informed consent. The expression levels of SP and NK1R were evaluated by immunohistochemical staining of tissue microarrays (TMAs) (Shanghai Biochip Co., Ltd., Shanghai, People's Republic of China). The TMAs contain a total of 267 formalin-fixed, paraffin-embedded tumor samples from 267 CRC patients, while 267 adjacent normal tissues were derived as corresponding controls. All patients had follow-up records for $>5$ years, and the survival time was calculated from the date of surgery to the date of death or follow-up deadline.

\section{Immunohistochemistry analysis}

TMA sections were then used for the immunohistochemical staining as described previously. ${ }^{24}$ Briefly, TMA sections were deparaffinized with xylene and then dehydrated through descending grades of ethanol to deionized water according to standard procedures. After antigen retrieval with the appropriate buffer ( $0.01 \mathrm{M}$ citrate buffer, $\mathrm{pH} 6.0$, high to boiling) for 3 minutes, the sections were cooled at room temperature. Endogenous peroxidase was blocked with 3\% (v/v) aqueous hydrogen peroxide for 10 minutes, followed by incubation with normal serum for 20 minutes to reduce nonspecific binding. Then, the sections were incubated with a primary antibody against SP (1:100, SC9758; Santa Cruz Biotechnology Inc., Dallas, TX, USA) or an antibody against NK1R (1:250, SC14116; Santa Cruz Biotechnology Inc) overnight at $4^{\circ} \mathrm{C}$. Negative controls were included, and steps were carried out with PBS. Subsequently, TMA sections were incubated with biotin-labeled secondary antibody (Thermo Fisher Scientific, Waltham, MA, USA) for 20 minutes at room temperature, followed by incubation with streptavidinbiotinylated horseradish peroxidase-conjugated antibody (Thermo Fisher Scientific) for another 20 minutes. Finally, the sections were stained with 3,3-diaminobenzidine and lightly counterstained with Mayer's hematoxylin, dehydrated, and mounted with resinous mounting medium.

\section{Evaluation of immunohistochemical staining}

The degree of immunostaining was examined semiquantitatively and scored under a light microscope by two independent expert pathologists without prior knowledge of the clinical data. Expression level of SP and NK1R was based on the Allred 8-unit system using the combination of an intensity 
staining score and a proportion score of stained tumor cells. The staining intensity was scored as follows: 0 (no staining), 1 (weak staining, light yellow), 2 (moderate staining, yellow brown), and 3 (strong staining, brown). Proportion of stained tumor cells was scored according to the proportion of positively staining tumor cells: 0 for $<5 \%$ positive tumor cells; 1 for $6 \%-25 \%$ positive tumor cells; 2 for $26 \%-50 \%$ positive tumor cells; and 3 for $>51 \%$ positive tumor cells. For final evaluation, a total score of $\leq 4$ was regarded as low expression for SP or NK1R, and a score of $>5$ was used to define tumors with high expression of SP or NK1R.

\section{Statistical analysis}

All statistical analyses were performed using the SPSS (Version 13.0; SPSS Inc., Chicago, IL, USA). To evaluate the associations between the expression of SP and NK1R and the clinicopathological features of the CRC patients, chi-square $\left(\chi^{2}\right)$ test or Fisher's exact test was used. Univariate survival analysis was performed using the Kaplan-Meier method accompanying log rank test. Cox proportional hazards regression model was used to perform multivariate survival analysis to estimate the predictors related to prognosis. Correlation between SP, NK1R protein expression, and clinicopathological parameters was estimated using Spearman correlation method. All $P$-values were two sided, and a $P$-value $<0.05$ was considered statistically significant.

\section{Results}

\section{Expression of SP and NKIR in CRC and adjacent normal tissues}

The immunostaining for SP was predominant in the cytoplasm (Figure 1). SP was highly expressed in 183 (68.5\%) of the 267 patients with CRC, which was significantly higher than the expression observed in the adjacent normal tissues $(2.2 \%$, $6 / 267, P<0.001)$. The immunostaining for NK1R was also mainly located in the cytoplasm. High expression levels of NK1R were detected in 199 (74.5\%) of the 267 patients with CRC, significantly higher than that in the adjacent normal tissues $(5.6 \%, 15 / 267, P<0.001)$. The scoring of immunohistochemical staining for SP and NK1R is shown in Figure 2.

\section{Patient characteristics and association of SP and NKIR expression with clinicopathological features of CRC}

The patient cohort consisted of 142 males and 125 females, with a median age of 67 years (range 24-91) at the time of surgery. High expression of SP in CRC has no significant association with age, sex, distance metastasis, pathology grading, and TNM (tumor node metastasis) stage but was significantly associated with lymph node metastasis (Table 1). In all, 84.9\% (90/106) of CRC patients with lymph node metastasis were detected with high expression of SP, which was higher than that without lymph node metastasis $\left(57.8 \%, 93 / 161, \chi^{2}=21.837, P<0.001\right)$. The Spearman correlation coefficient of high SP expression with lymph node metastasis was $0.286(P<0.001)$.

High NK1R expression in CRC had no significant association with age, sex, distance metastasis, and pathology grading but was significantly related to lymph node metastasis and TNM stage (Table 2). High NK1R expression was detected in $83.0 \%(88 / 106)$ of CRC cases with lymph node metastasis, which was significantly higher than the expression in patients without lymph node metastasis $(68.9 \%, 111 / 161$, $\left.\chi^{2}=6.671, P=0.010\right)$. High NK1R expression was detected in $77.2 \%(176 / 228)$ of CRC patients with TNM stage III + IV, which was higher than the expression in patients with TNM stage I + II $\left(59.0 \%, 23 / 39, \chi^{2}=5.823, P=0.019\right)$. The Spearman correlation coefficients of high NK1R expression with lymph node metastasis and TNM stage were 0.158 and 0.148 $(P<0.05)$, respectively.

\section{Correlation of SP and NKIR expression with prognosis}

Kaplan-Meier survival analysis showed that the 3- and 5 -year cumulative survival rates for patients with high expression of SP were $43.8 \%$ and $22.8 \%$, while for those with low SP expression, the survival rates were $82.5 \%$ and $75.0 \%$, respectively (Figure 3 ). The follow-up time for the patients was in August 2013. The mean survival time for CRC patients with high SP expression was $43.53 \pm 2.42$ months, and the survival time for patients with low SP expression was $79.98 \pm 1.60$ months. Clearly, CRC patients with high expression levels of SP have a poorer prognosis (log rank test, $\left.\chi^{2}=68.010, P<0.001\right)$. The 3 - and 5-year cumulative survival rates for patients with high expression of NK1R were $51.6 \%$ and $31.9 \%$, while for those with a low NK1R expression, the survival rates were $65.5 \%$ and $56.4 \%$, respectively. The mean survival time for CRC patients with high NK1R expression was $52.85 \pm 2.41$ months and that for patients with low NK1R expression was $70.38 \pm 3.47$ months. CRC patients with high expression of NK1 $\mathrm{R}$ have a poorer prognosis (log rank test, $\left.\chi^{2}=11.333, P=0.001\right)$. Multivariate analysis using Cox regression model showed that survival rate was independently correlated with lymph node metastasis, distance metastasis, and SP expression $(P<0.05$; Table 3$)$. 

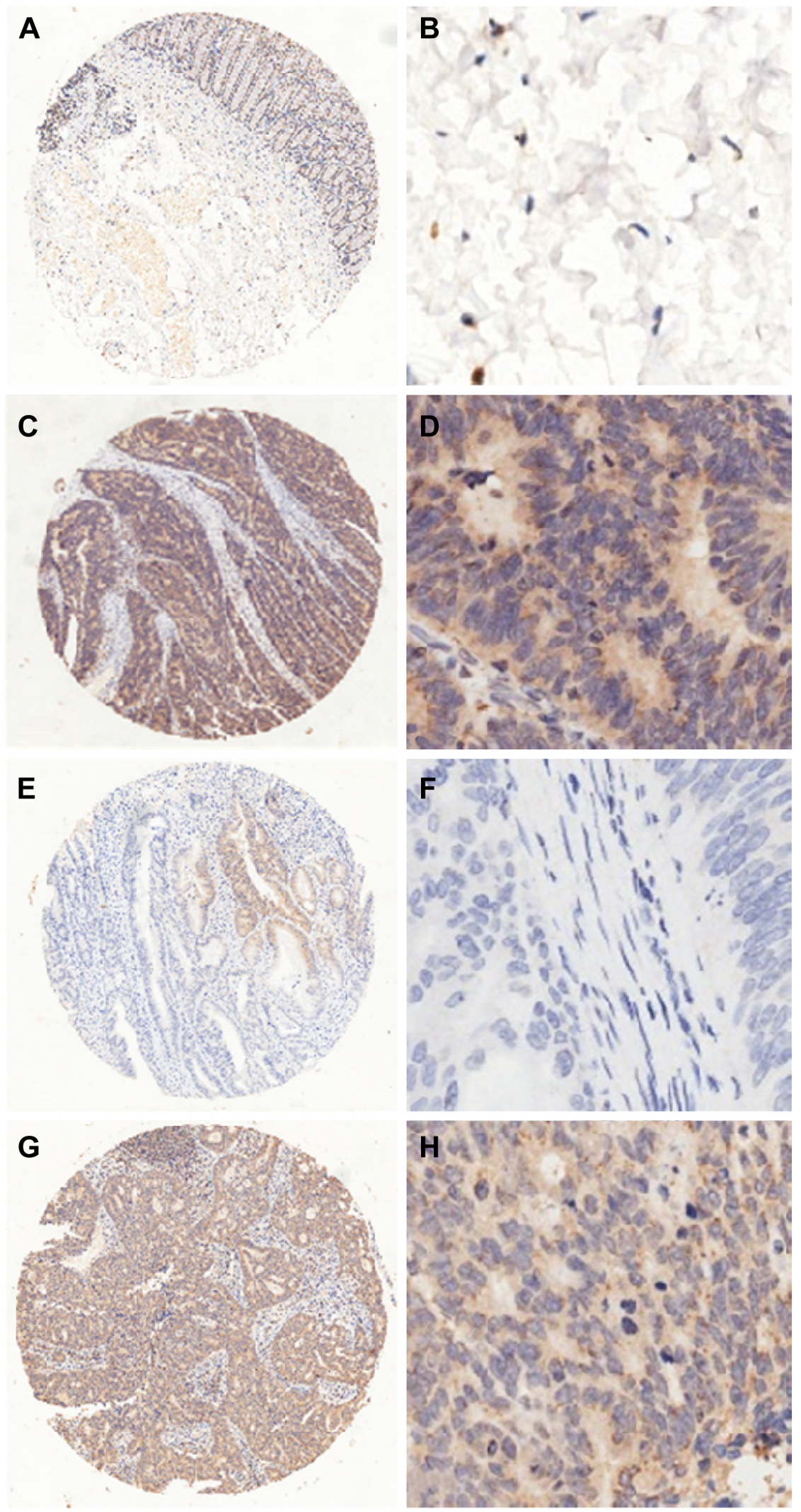

Figure I Immunohistochemical staining of SP and NKIR in CRC and normal tissue.

Notes: (A and B) Low expression of SP in normal colon tissues adjacent to cancerous tissues. (C and D) High expression of SP in a tumor sample and positive staining, primarily in the cytoplasm. (E and $\mathbf{F}$ ) Low expression of NKIR in adjacent normal colon tissues. ( $\mathbf{G}$ and $\mathbf{H}$ ) High expression of NKIR in tumor sample and positive staining, mainly in the cytoplasm. (A, C, E, and G) Original magnification 200×; (B, D, F, and H) original magnification 800x.

Abbreviations: NKIR, neurokinin-I receptor; CRC, colorectal cancer; SP, substance P. 


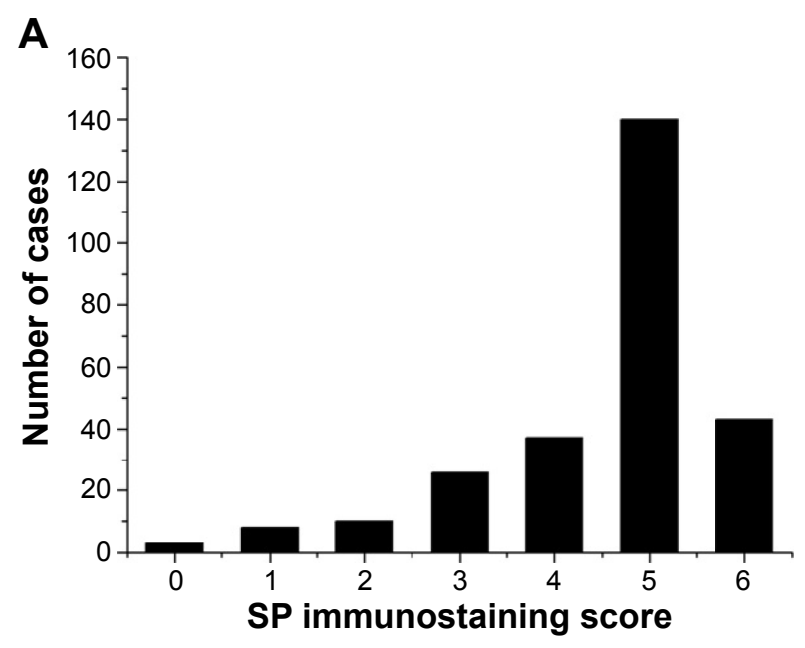

Figure 2 The scoring of immunohistochemical staining for SP (A) and NKIR (B). Abbreviations: SP, substance P; NKIR, neurokinin-I receptor.

\section{Association between SP and NKIR expression levels in CRC}

Our results indicate a high correlation between SP and NK1R expression in CRC. Of the 183 patients with high expression of SP, $155(84.7 \%)$ also had a high expression of NK1R. The correlation between the expression of SP and NK1R in patients with CRC was statistically significant $(r=0.419$, $P<0.001$, Spearman's $\rho$-test).

\section{Discussion}

Links between cancer and inflammation were made on the basis of observations that the tumors often arose at the sites

Table I Relationship of SP expression with clinicopathological features of CRC

\begin{tabular}{|c|c|c|c|c|}
\hline Parameters & $\begin{array}{l}\text { Strong } \\
\text { expression }\end{array}$ & $\begin{array}{l}\text { Weak or no } \\
\text { expression }\end{array}$ & $\chi^{2}$ & $P$-value \\
\hline \multicolumn{5}{|l|}{ Age } \\
\hline$<67$ years & 85 & 38 & 0.034 & 0.895 \\
\hline$\geq 67$ years & 98 & 46 & & \\
\hline \multicolumn{5}{|l|}{ Sex } \\
\hline Male & 96 & 46 & 0.123 & 0.792 \\
\hline Female & 87 & 38 & & \\
\hline \multicolumn{5}{|c|}{ Distance metastasis } \\
\hline Yes & 7 & I & 1.375 & 0.290 \\
\hline No & 176 & 83 & & \\
\hline \multicolumn{5}{|c|}{ Lymph nodes metastasis } \\
\hline Positive & 90 & 16 & 21.837 & $<0.001$ \\
\hline Negative & 93 & 68 & & \\
\hline \multicolumn{5}{|l|}{ TNM stage } \\
\hline$I+I I$ & 24 & 15 & 1.308 & 0.352 \\
\hline III + IV & 159 & 69 & & \\
\hline \multicolumn{5}{|c|}{ Pathology grading } \\
\hline I & 12 & 9 & 4.735 & 0.094 \\
\hline II & 112 & 58 & & \\
\hline III & 59 & 17 & & \\
\hline
\end{tabular}

Abbreviations: SP, substance P; CRC, colorectal cancer; TNM, tumor node metastasis.

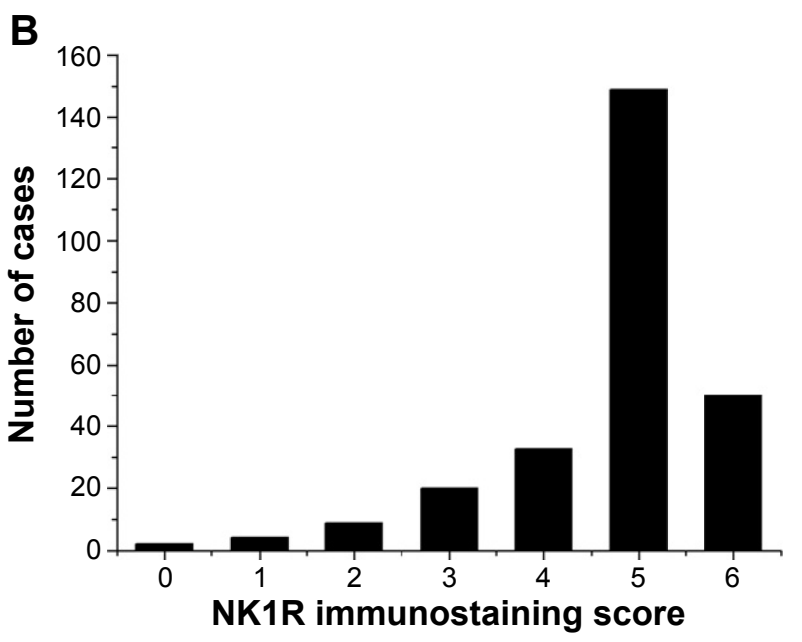

of chronic inflammation and the presence of inflammatory cells in the biopsy samples from tumor. ${ }^{5}$ It is widely accepted that the inflammation mediators and cellular effectors are important constituents of tumor microenvironments. ${ }^{25}$ Recently, the effects of the inflammatory response on the development of neoplasia were demonstrated in various types of cancers such as breast, endometrial, ovarian, and colon tumors. ${ }^{5}$ It has been observed that patients with inflammatory bowel diseases are at an increased risk of neoplasia development and progression to CRC. Inflammation is considered to be an important risk factor for the development of CRC. ${ }^{26}$

Table 2 Relationship of NKIR expression with clinicopathological features of CRC

\begin{tabular}{|c|c|c|c|c|}
\hline Parameters & $\begin{array}{l}\text { Strong } \\
\text { expression }\end{array}$ & $\begin{array}{l}\text { Weak or no } \\
\text { expression }\end{array}$ & $\chi^{2}$ & $P$-value \\
\hline \multicolumn{5}{|l|}{ Age } \\
\hline$<67$ years & 96 & 27 & 1.486 & 0.260 \\
\hline$\geq 67$ years & 103 & 41 & & \\
\hline \multicolumn{5}{|l|}{ Sex } \\
\hline Male & 112 & 30 & 3.012 & 0.092 \\
\hline Female & 87 & 38 & & \\
\hline \multicolumn{5}{|c|}{ Distance metastasis } \\
\hline Yes & 7 & 1 & 0.731 & 0.469 \\
\hline No & 192 & 67 & & \\
\hline \multicolumn{5}{|c|}{ Lymph nodes metastasis } \\
\hline Positive & 88 & 18 & 6.671 & 0.010 \\
\hline Negative & III & 50 & & \\
\hline \multicolumn{5}{|l|}{ TNM stage } \\
\hline$I+I I$ & 23 & 16 & 5.823 & 0.019 \\
\hline $\mathrm{III}+\mathrm{IV}$ & 176 & 52 & & \\
\hline \multicolumn{5}{|c|}{ Pathology grading } \\
\hline 1 & 14 & 7 & 1.208 & 0.566 \\
\hline II & 130 & 40 & & \\
\hline III & 55 & 21 & & \\
\hline
\end{tabular}

Abbreviations: NKIR, neurokinin-I receptor; CRC, colorectal cancer; TNM, tumor node metastasis. 


\section{A}

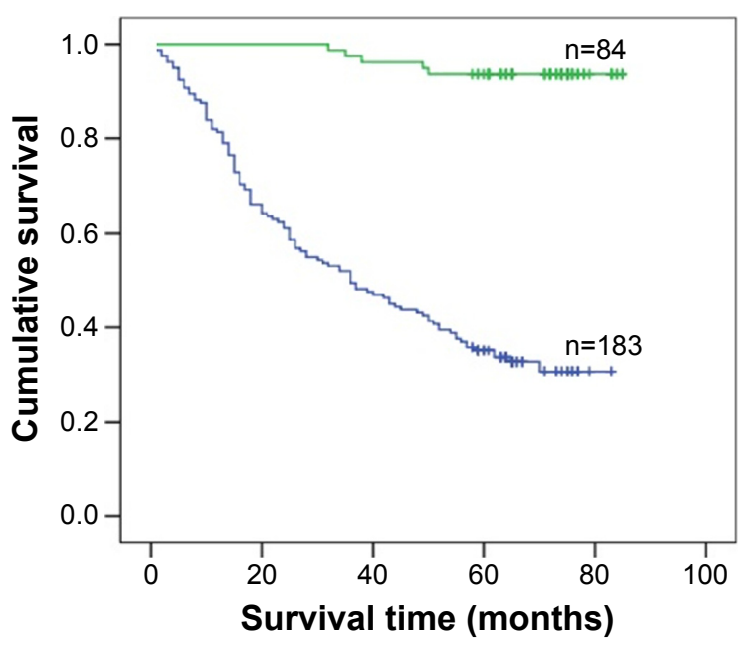

\begin{tabular}{|l|}
\hline SP expression in CRC \\
$\neg$ Strong expression \\
$\neg$ Weak or no expression \\
+ Strong expression-censored \\
+ Weak or no expression-censored
\end{tabular}

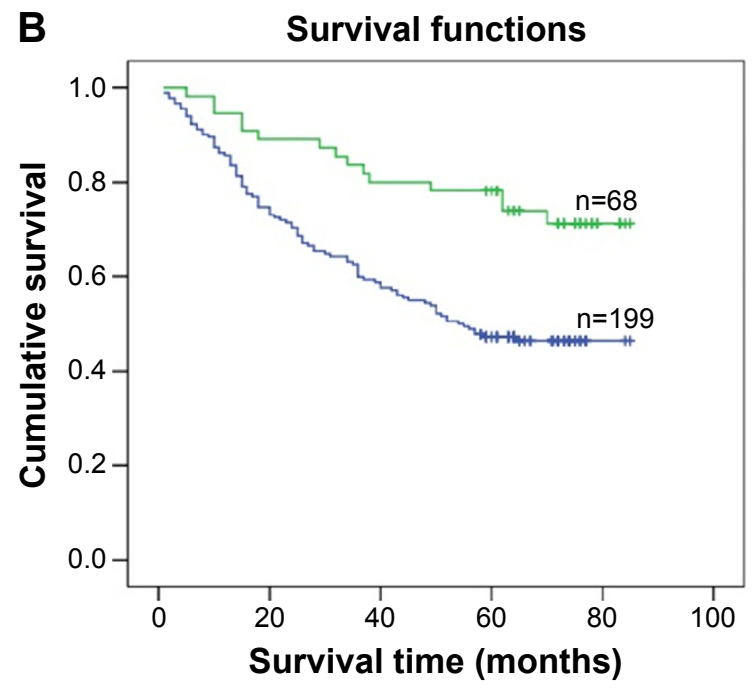

NK1R expression in CRC

$\neg$ Strong expression

$\neg$ Weak or no expression

+ Strong expression-censored

+ Weak or no expression-censored

Figure 3 Kaplan-Meier survival analysis for CRC patients with SP (A) and NKIR (B) expression. Abbreviations: CRC, colorectal cancer; SP, substance P; NKIR, neurokinin-I receptor.

SP is secreted by nerve fibers and inflammatory cells such as macrophages, lymphocytes, and eosinophils and has a proinflammatory effect and participates in inflammatory immune responses. The link between inflammation and cancer could be explained through SP-centered models. ${ }^{20}$ Numerous studies have reported the involvement of the SP/NK1R system in cancer. ${ }^{27} \mathrm{SP}$ can induce mitosis in normal and tumor cells and protects tumor cells from apoptosis. ${ }^{21,28,29}$ Binding of SP to NK1R triggers the release of secondary messengers (cAMP) triggering a number of effector mechanisms involved in the regulation of cellular functions. ${ }^{30}$ It has previously been reported that NK1R activation can induce the phosphorylation of Akt and MAPK family members, which subsequently lead to the activation of different transcription factors regulating the expression of respective target genes. ${ }^{30-34}$ The regulatory mechanisms stimulate DNA synthesis and/or cytokine secretion and mediate the antiapoptotic effect. ${ }^{35}$ However, the function of SP-NK1R pathway in the regulation of inflammation-induced pathogenesis in CRC is poorly understood.

In this study, we evaluated the expression levels of SP and NK1R in CRC and showed that the expression levels can be implicated in the prediction of CRC prognosis. SP expression was upregulated in CRC as compared with that in adjacent normal tissues, and elevated SP expression was significantly associated with lymph node metastasis. This result is consistent with the findings associating the role of SP in the migration of tumor cells leading to metastasis, which is a major problem in the treatment of tumors. Moreover, it has been recently reported that the cerebral metastases result

Table 3 Cox regression analysis of the clinicopathological parameters in colon cancer patients

\begin{tabular}{lllll}
\hline Parameters & Coefficient & Hazard ratio (HR) & 95\% Cl for HR & P-value \\
\hline Age & -0.004 & 0.996 & $0.979-1.014$ & 0.665 \\
Sex & -0.235 & 0.791 & $0.528-1.184$ & 0.254 \\
Distance metastasis & 1.656 & 5.236 & $2.128-12.880$ & $<0.001$ \\
Lymph nodes metastasis & 0.463 & 1.589 & $1.028-2.455$ & 0.037 \\
TNM stage & 0.677 & 1.968 & $0.776-4.993$ & 0.154 \\
Pathology grading & 0.332 & 1.394 & $0.956-2.032$ & 0.084 \\
SP expression & 2.922 & 18.584 & $7.069-48.858$ & $<0.001$ \\
NKIR expression & $-0.35 I$ & 0.704 & $0.379-1.309$ & 0.268 \\
\hline
\end{tabular}

Abbreviations: SP, substance P; NKIR, neurokinin-I receptor; TNM, tumor node metastasis. 
from SP-mediated process. ${ }^{36}$ Our results indicate that SP is a proinflammatory modulator, and its expression levels are linked to different stages of CRC development.

It is well known that malignant tissues have high expression levels of NK1R, and a large percentage of malignant tumor cell phenotypes show increased NK1R expression. ${ }^{37}$ Similarly, we found that the expression of NK1R was upregulated in CRC as compared with that in adjacent normal tissues, and elevated expression was significantly associated with lymph node metastasis and TNM stage. Besides, our results showed that patients with high expression of SP also had a high rate of upregulated expression of NK1R in CRC. Our results strongly indicate that SP/NK1R system can be used as a mark predicting the invasion and metastasis of CRC. The results also indicate the involvement of inflammatory processes in the tumor microenvironment.

A strong tumor-associated inflammatory reaction analogous to a wound-healing response can be initiated by cancer therapy. ${ }^{38}$ The outcome of therapy-induced inflammatory response can have tumor-promoting functions similar to necrosis, which accompanies rapid tumor growth. ${ }^{39,40}$ As a result, the inflammation may also play an important role in the prognosis of CRC. Turner et $\mathrm{al}^{41}$ examined the relationship between tachykinin concentrations and survival of tumor patients and indicated that tachykinins in tumor patients could be used as a strong prognostic indicator. Our study also shows that CRC patients with high expression of SP or NK1R have a poor prognosis. However, one of the drawbacks of our study is the use of TMA, where the amount of tissue analyzed using this technique is limited and may not be representative of the whole specimen. This may pose a significant problem in malignant epithelial tumors where there is a high degree of intratumoral heterogeneity. Therefore, further analysis about the association between SP or NK1R and CRC development is still to be studied.

\section{Conclusion}

Upregulated expression of SP-NK1R may play a crucial role in CRC progression; moreover, SP-NK1R expression may also be used as a predictor for prognosis of CRC. Based on these findings, regulation of SP and NK1R expression and/or the inhibition of the potential signaling pathways associated with SP and NK1R could be used to develop novel therapeutic strategies for CRC in the future.

\section{Acknowledgments}

This work was supported by the National Science Foundation of China (nos 81201783, 81372463, and 81472210), Zhejiang
Provincial Natural Science Foundation of China (nos LY15H160051, LY14H160041, and LY13H080005), Funds of Science Technology Department of Zhejiang Province (no 2014C37101), Zhejiang Province Bureau of Health (no 2015ZA009), and Open Fund of Zhejiang Provincial Top Key Discipline of Biology.

\section{Disclosure}

The authors report no conflicts of interest in this work.

\section{References}

1. Peto J. Cancer epidemiology in the last century and the next decade. Nature. 2001;411(6835):390-395.

2. Ferlay J, Shin HR, Bray F, Forman D, Mathers C, Parkin DM. Estimates of worldwide burden of cancer in 2008: GLOBOCAN 2008. Int J Cancer. 2010;127(12):2893-2917.

3. Chen $\mathrm{W}$, Zheng $\mathrm{R}$, Zhang $\mathrm{S}$, et al. Report of incidence and mortality in China cancer registries, 2009. Chin J Cancer Res. 2013;25(1):10-21.

4. Federico A, Morgillo F, Tuccillo C, Ciardiello F, Loguercio C. Chronic inflammation and oxidative stress in human carcinogenesis. Int $J$ Cancer. 2007;121(11):2381-2386.

5. Morrison WB. Inflammation and cancer: a comparative view. $J$ Vet Intern Med. 2012;26(1):18-31.

6. Patacchini R, Maggi CA. Peripheral tachykinin receptors as targets for new drugs. Eur J Pharmacol. 2001;429(1-3):13-21.

7. Vanden Broeck J, Torfs H, Poels J, et al. Tachykinin-like peptides and their receptors. A review. Ann N Y Acad Sci. 1999;897:374-387.

8. Ho WZ, Lai JP, Zhu XH, Uvaydova M, Douglas SD. Human monocytes and macrophages express substance $\mathrm{P}$ and neurokinin-1 receptor. J Immunol. 1997;159(11):5654-5660.

9. Pernow B. Substance P. Pharmacol Rev. 1983;35(2):85-141.

10. Mantyh PW. Substance $P$ and the inflammatory and immune response. Ann N Y Acad Sci. 1991;632:263-271.

11. Harrison S, Geppetti P. Substance P. Int J Biochem Cell Biol. 2001; 33(6):555-576.

12. Quartara L, Maggi CA. The tachykinin NK1 receptor. Part II: distribution and pathophysiological roles. Neuropeptides. 1998;32(1):1-49.

13. Munoz M, Rosso M, Robles-Frias MJ, et al. The NK-1 receptor is expressed in human melanoma and is involved in the antitumor action of the NK-1 receptor antagonist aprepitant on melanoma cell lines. Lab Invest. 2010;90(8):1259-1269.

14. Esteban F, Gonzalez-Moles MA, Castro D, et al. Expression of substance $\mathrm{P}$ and neurokinin-1-receptor in laryngeal cancer: linking chronic inflammation to cancer promotion and progression. Histopathology. 2009; 54(2):258-260.

15. Rosso M, Robles-Frias MJ, Covenas R, Salinas-Martin MV, Munoz M. The NK-1 receptor is expressed in human primary gastric and colon adenocarcinomas and is involved in the antitumor action of L-733,060 and the mitogenic action of substance $\mathrm{P}$ on human gastrointestinal cancer cell lines. Tumour Biol. 2008;29(4):245-254.

16. Hennig IM, Laissue JA, Horisberger U, Reubi JC. Substance-P receptors in human primary neoplasms: tumoral and vascular localization. Int J Cancer. 1995;61(6):786-792.

17. Schulz S, Stumm R, Rocken C, Mawrin C, Schulz S. Immunolocalization of full-length NK1 tachykinin receptors in human tumors. J Histochem Cytochem. 2006;54(9):1015-1020.

18. Lazarczyk M, Matyja E, Lipkowski A. Substance P and its receptors - a potential target for novel medicines in malignant brain tumour therapies (mini-review). Folia Neuropathol. 2007;45(3):99-107.

19. Guha S, Eibl G, Kisfalvi K, et al. Broad-spectrum G protein-coupled receptor antagonist, [D-Arg1,D-Trp5,7,9,Leu11]SP: a dual inhibitor of growth and angiogenesis in pancreatic cancer. Cancer Res. 2005;65(7):2738-2745. 
20. Esteban F, Munoz M, Gonzalez-Moles MA, Rosso M. A role for substance $\mathrm{P}$ in cancer promotion and progression: a mechanism to counteract intracellular death signals following oncogene activation or DNA damage. Cancer Metastasis Rev. 2006;25(1):137-145.

21. Munoz M, Rosso M, Covenas R. A new frontier in the treatment of cancer: NK-1 receptor antagonists. Curr Med Chem. 2010;17(6):504-516.

22. Munoz M, Rosso M. The NK-1 receptor antagonist aprepitant as a broad spectrum antitumor drug. Invest New Drugs. 2010;28(2):187-193.

23. Fang WJ, Fu CY, Chen XG, et al. Neurokinin-2 receptor polymorphism predicts lymph node metastasis in colorectal cancer patients. Oncol Lett. 2015;9(5):2003-2006.

24. Liu FL, Ye PJ, Bi TN, et al. COLORECTAL polymeric immunoglobulin receptor expression is correlated with hepatic metastasis and poor prognosis in colon carcinoma patients with hepatic metastasis. Hepatogastroenterology. 2014;61(131):652-659.

25. Mantovani A, Allavena P, Sica A, Balkwill F. Cancer-related inflammation. Nature. 2008;454(7203):436-444.

26. Klampfer L. Cytokines, inflammation and colon cancer. Curr Cancer Drug Targets. 2011;11(4):451-464.

27. Munoz M, Rosso M, Covenas R. The NK-1 receptor: a new target in cancer therapy. Curr Drug Targets. 2011;12(6):909-921.

28. DeFea KA, Vaughn ZD, O’Bryan EM, Nishijima D, Dery O, Bunnett NW. The proliferative and antiapoptotic effects of substance $P$ are facilitated by formation of a beta-arrestin-dependent scaffolding complex. Proc Natl Acad Sci U S A. 2000;97(20):11086-11091.

29. Feng F, Yang J, Tong L, et al. Substance P immunoreactive nerve fibres are related to gastric cancer differentiation status and could promote proliferation and migration of gastric cancer cells. Cell Biol Int. 2011;35(6):623-629.

30. Mitsuhashi M, Ohashi Y, Shichijo S, et al. Multiple intracellular signaling pathways of the neuropeptide substance P receptor. J Neurosci Res. 1992;32(3):437-443.

31. Luo W, Sharif TR, Sharif M. Substance P-induced mitogenesis in human astrocytoma cells correlates with activation of the mitogen-activated protein kinase signaling pathway. Cancer Res. 1996;56(21):4983-4991.
32. Akazawa T, Kwatra SG, Goldsmith LE, et al. A constitutively active form of neurokinin 1 receptor and neurokinin 1 receptor-mediated apoptosis in glioblastomas. J Neurochem. 2009;109(4):1079-1086.

33. Lieb K, Fiebich BL, Berger M, Bauer J, Schulze-Osthoff K. The neuropeptide substance $\mathrm{P}$ activates transcription factor NF-kappa B and kappa B-dependent gene expression in human astrocytoma cells. J Immunol. 1997;159(10):4952-4958.

34. Christian C, Gilbert M, Payan DG. Stimulation of transcriptional regulatory activity by substance P. Neuroimmunomodulation. 1994;1(3): 159-164.

35. Koon HW, Zhao D, Zhan Y, Moyer MP, Pothoulakis C. Substance P mediates antiapoptotic responses in human colonocytes by Akt activation. Proc Natl Acad Sci U S A. 2007;104(6):2013-2018.

36. Lewis KM, Harford-Wright E, Vink R, Nimmo AJ, Ghabriel MN. Walker 256 tumour cells increase substance P immunoreactivity locally and modify the properties of the blood-brain barrier during extravasation and brain invasion. Clin Exp Metastasis. 2013;30(1):1-12.

37. Friess H, Zhu Z, Liard V, et al. Neurokinin-1 receptor expression and its potential effects on tumor growth in human pancreatic cancer. $L a b$ Invest. 2003;83(5):731-742.

38. Zong WX, Thompson CB. Necrotic death as a cell fate. Genes Dev. 2006;20(1):1-15.

39. Ammirante M, Luo JL, Grivennikov S, Nedospasov S, Karin M. B-cellderived lymphotoxin promotes castration-resistant prostate cancer. Nature. 2010;464(7286):302-305.

40. Vakkila J, Lotze MT. Inflammation and necrosis promote tumour growth. Nat Rev Immunol. 2004;4(8):641-648.

41. Turner GB, Johnston BT, McCance DR, et al. Circulating markers of prognosis and response to treatment in patients with midgut carcinoid tumours. Gut. 2006;55(11):1586-1591.
OncoTargets and Therapy

\section{Publish your work in this journal}

OncoTargets and Therapy is an international, peer-reviewed, open access journal focusing on the pathological basis of all cancers, potential targets for therapy and treatment protocols employed to improve the management of cancer patients. The journal also focuses on the impact of management programs and new therapeutic agents and protocols on

\section{Dovepress}

patient perspectives such as quality of life, adherence and satisfaction The manuscript management system is completely online and includes a very quick and fair peer-review system, which is all easy to use. Visit http://www.dovepress.com/testimonials.php to read real quotes from published authors. 